\title{
Patria, razza e civiltà. Le istruzioni per un'emigrazione di successo nei manuali scolastici di Geografia italiani tra fine ' 800 e inizio ' 900
}

Pátria, raça e civilização. As instruções para uma emigração de sucesso nos manuais de Geografia italianos entre o final do Século XIX e o início do XX

Homeland, race and civilization. The instructions for a successful emigration in the italian Geography textbooks between the end of ' 800 and the beginning of the ' 900

Patria, raza y civilización. Las instrucciones para una emigración de éxito en los manuales de Geografía italianos entre el final del 800 y el inicio del 900

\author{
Paolo Bianchini \\ Università degli Studi di Torino (Italia) \\ https://orcid.org/0000-0002-2938-0516 \\ paolo.bianchini@unito.it
}

\section{RIASSUNTO}

Il saggio intende illustrare le modalità in cui l'emigrazione e la colonizzazione erano presentate e giustificate nei manuali di geografia italiani nei decenni a cavallo tra XIX e XX secolo. A tal fine sarà analizzata una serie di libri scolastici in uso nelle scuole primarie e secondarie. Molto frequentemente, infatti, i temi dell'emigrazione e della colonizzazione erano trattati ampiamente a scuola, sia perché l'emigrazione era all'epoca un fenomeno di massa che riguardava pressoché tutte le famiglie sia perché l'Italia, recentemente unificata, era alla ricerca di una sua dimensione internazionale per mezzo di una campagna di conquista territoriale e culturale che si estendeva ben oltre il Mediterraneo sino a raggiungere i Paesi al di là dell'Oceano Atlantico. L'obiettivo è analizzare il ruolo della scuola e dell'editoria scolastica nella costruzione di concetti centrali nella vita degli individui, come patria, razza e civiltà, che ancora oggi risentono fortemente delle immagini e degli insegnamenti trasmessi nei secoli passati.

Parole chiave: Manuale scolastico; Emigrazione; Sud America. 


\section{RESUMO}

O artigo pretende analisar as formas como a emigração e a colonização foram apresentadas e justificadas nos manuais de geografia italianos durante os decênios finais do século XIX e primeiros do XX. Para este fim é analisada uma série de livros escolares utilizados nas escolas primárias e secundárias. Os temas da emigração e da colonização eram amplamente tratados nas escolas, tanto porque a emigração era na época um fenômeno de massa que afetava quase todas as famílias italianas quanto porque a Itália, recentemente unida, estava em busca de uma dimensão internacional através de uma campanha de conquista territorial e cultural que se estendeu muito além do Mediterrâneo para alcançar os países além do Oceano Atlântico. $\mathrm{O}$ objetivo final é analisar o papel da escola e da editoria escolar na construção de conceitos centrais na vida dos indivíduos, como pátria, raça e civilização, que ainda hoje são fortemente influenciados pelas imagens e ensinamentos constituídos nos séculos passados.

Palavras-chave: Manual escolar; Emigração; América do Sul.

\section{ABSTRACT}

The essay aims to illustrate the ways in which emigration and colonization were presented and justified in Italian geography manuals in the decades between the nineteenth and twentieth centuries. To this end, a series of schoolbooks in use in primary and secondary schools will be analysed. Very frequently, in fact, the issues of emigration and colonization were widely treated at school, both because emigration was at the time a mass phenomenon that affected almost all families and because Italy, recently unified, was constructing its international dimension by means of a campaign of territorial and cultural conquest extended far beyond the Mediterranean area to reach the countries beyond the Atlantic Ocean.

The goal is to analyse the role of school and school publishing in the construction of some central concepts in the life of individuals, such as homeland, race and civilization, which still today are strongly affected by the images and the instructions transmitted in the past centuries.

Key words: Textbook; Emigration; South America.

\section{RESUMEN}

El artículo pretende analizar las formas como la emigración y la colonización fueron presentadas y justificadas en los manuales de geografía italianos durante los decenios finales del siglo XIX y primeros del XX. Para este fin se analiza una serie de libros escolares utilizados en las escuelas primarias y secundarias. Los temas de la emigración y de la colonización eran ampliamente tratados en las escuelas, tanto porque la emigración era en la época un fenómeno de masa que afectaba a casi todas las familias italianas, ya que Italia, recientemente unida, estaba en busca de una dimensión internacional a través de una campaña logro territorial y cultural que se ha extendido mucho más allá del Mediterráneo hasta llegar a los países a través del Océano Atlántico. El objetivo final es analizar el papel de la escuela y de la editorial escolar en la construcción de conceptos centrales en la vida de los individuos, como patria, raza y civilización, que aún hoy están fuertemente influenciados por las imágenes y enseñanzas constituidas en los siglos pasados.

Palabras clave: Manual escolar; Emigración; América del Sur. 


\section{Una questione sociale, politica e culturale di portata continentale}

Grazie agli studi che si sono moltiplicati negli ultimi decenni in molti ambiti differenti delle scienze umane, oggi conosciamo in maniera molto più approfondita la realtà dei numerosissimi italiani e, più in generale degli europei che, alla fine dell'Ottocento, lasciarono la madrepatria in cerca di fortuna o di condizioni di vita più sicure. Si trattò di un fenomeno complessissimo, con cause e ricadute molteplici e solo in parte comprensibili anche a distanza di un secolo circa. Anche gli osservatori coevi faticarono a dare una lettura comprensibile e realistica all'emigrazione.

Gli stessi governi che si succedettero alla guida dell'Italia nei decenni successivi all'unità impiegarono non poco tempo a cogliere l'enormità del fenomeno, con le sue ricadute sociali e politiche, oltre che economiche. Dare un senso compiuto al fenomeno migratorio, che riguardò non solo l'Italia, ma buona parte dell'Europa tra la fine dell'Ottocento e il secondo dopoguerra fu operazione non semplice e soprattutto non univoca anche per gli osservatori più esperti e distaccati.

Economisti, sociologi, politologi diedero vita a un dibattito molto acceso circa le molteplici valenze che la fuoriuscita massiccia di popolazione verso altri Stati e altri continenti assumeva per la nazione che abbandonavano. Emorragia per alcuni, occasione di rilancio economico per altri, merce di scambio e mezzo per estendere la propria influenza politica per altri ancora: non diversamente da quanto avviene ancora oggi, l'emigrazione raramente è stata guardata dai contemporanei con gli occhi dell'emigrante.

Poiché il fenomeno migratorio assunse dimensioni quasi di massa in Paesi come l'Italia, e moltissime famiglie si confrontarono con la scelta di emigrare e sognare un futuro migliore o restare e accontentarsi di ciò che avevano, ho pensato di verificare se e come il tema veniva trattato in una fonte per sua natura umile, ma capillarmente diffusa anche tra gli strati più umili della popolazione come il manuale scolastico.

È vero che chi decideva di partire era spesso (anche se non sempre) dotato di scarsa cultura, oltre che in situazione di tale difficoltà per cui abbandonare il suolo natio e recarsi in una terra straniera rappresentava una scelta quasi incosciente, ma senza alternative. Tuttavia, è altrettanto certo che, sin dall'Unità d'Italia, specialmente nelle regioni del Nord, la popolazione istruita aumentò gradualmente e molti erano coloro che, magari senza terminare il percorso formativo, frequentavano almeno i primi anni delle scuole primarie.

Per questi motivi, ho esaminato una selezione di libri di testo di geografia per le scuole elementari e secondarie prodotti in Italia in un arco di tempo che va dal 1870 al 1925 . La scelta è ricaduta in prima battuta sui libri che, alla luce delle nostre conoscenze attuali ${ }^{1}$, sembrano essere stati più diffusi all'epoca, quindi, su quelli effettivamente reperibili on line $o$ nelle biblioteche nazionali. ${ }^{2}$

Il risultato, come spero di dimostrare nelle pagine che seguono, è che nei manuali di geografia spesso si faceva riferimento all'emigrazione, e le interpretazioni che i testi scolastici offrivano erano assai varie. L'emigrazione non è mai stata un evento che lasciasse neutrali, ma è sempre stata spiegata e interpretata alla luce di una serie più o meno complessa di fattori. Per illustrare ai loro giovani lettori che cos'è l'emigrazione e perché si emigrasse, gli autori

1 La selezione è stata compiuta a partire dalla banca dati sul manuale scolastico italiano Edisco (http://www.database-edisco.org:8081/). Oltre ai dati reperibili on line, mi sono servito delle ricerche di G. Bandini, I maestri e la geografia dopo l'unità d'Italia, in P. Bianchini (a cura di), Le origini delle materie. Discipline, programmi e manuali scolastici in Italia, Torino, SEI, 2010, pp. 111-138; Id. (a cura di), Manuali, sussidi e didattica della geografia. Una prospettiva storica, Firenze, Firenze University Press, 2012 ; C. Tremolada, La Geografia nei libri di testo per le scuole elementari (1859-1945), Tesi di dottorato, Università degli Studi di Milano, 1993.

${ }^{2}$ Il campione preso in esame è composto da 40 manuali. Non in tutti i testi sono trattati i temi qui presi in considerazione. Per questo motivo, le citazioni sono tratte da un numero più ristretto di libri. 
dei manuali non potevano esimersi dal fare riferimento alla situazione politica, sociale ed economica coeva, richiamando temi allora centrali nella cultura europea. In particolare, ogni volta che si trattava di emigrazione facevano capolino nelle pagine dei testi scolastici i concetti di nazione, patria, colonia e razza: proprio su questi temi concentrerò la mia attenzione nell'ultima parte di questo saggio, per provare a ricostruire un po' più distesamente l'orizzonte ideologico e valoriale attribuito nelle scuole italiane a un evento epocale quale l'emigrazione.

\section{L'emigrazione tra realtà e rappresentazione a scuola}

Nei decenni successivi all'unificazione, l'Italia sperimentò il pressante problema di "fare gli italiani" e la scuola in questo senso acquisì un ruolo importante nelle intenzioni dei governi che si succedettero alla guida del Paese. Inoltre, il regno dei Savoia si stava affacciando per la prima volta verso l'estero con mire coloniali, mentre stava conoscendo un boom demografico senza precedenti, con flussi migratori ingentissimi rivolti verso le altre nazioni europee e ancor più oltreoceano. Fu specialmente a cavallo del secolo che in Italia il fenomeno migratorio assunse proporzioni assai rilevanti. ${ }^{3}$

Per questi motivi, oltre il 90\% dei manuali analizzati trattano il tema dell'emigrazione. Si tratta di una percentuale alta, che desta maggior sorpresa soprattutto per quanto riguarda $\mathrm{i}$ libri destinati alle scuole primarie, quando, per un lettore odierno, il pubblico era davvero ancora troppo giovane per comprendere concretamente un tema tanto complesso. Non va dimenticato, però, che esso riguardava all'epoca quasi tutte le famiglie e che, dunque, i bambini ne avevano spesso una conoscenza diretta, anche se magari fumosa. Inoltre, non va trascurato il fatto che l'infanzia era precocemente adultizzata, specie nei contesti con condizioni socio-economiche difficili.

Un dato che non può non sorprendere, invece, è che, a differenza di quanto avveniva in altri Paesi europei, come la Francia, gli autori italiani non solo trattavano il tema, sottolineando il ruolo degli emigranti italiani nei paesi di destinazione, ma frequentemente fornivano anche indicazioni abbastanza dettagliate per una emigrazione di successo. ${ }^{4}$

Il Compendio di geografia generale di Luigi Giannitrapani, ad esempio, dedica un ampio paragrafo a spiegare le teorie dell'emigrazione. ${ }^{5}$ In linea con il pensiero neo-idealista coevo, lo studioso considera gli Stati come organismi viventi che nascono, si sviluppano, si modificano, decadono e talvolta si estinguono, condizionando così la vita del proprio popolo. Tali cambiamenti possono generare fenomeni demografici di notevole portata, come ad esempio l'emigrazione. Essa può essere determinata dall'aumento costante della popolazione di uno Stato, oppure perchè le offerte di lavoro non sono sufficienti a soddisfare le richieste di tutti i cittadini o il lavoro non è abbastanza remunerativo, ma può anche essere frutto di condizioni politiche ed economiche disagiate per una parte della popolazione.

\footnotetext{
${ }^{3}$ Il tema è stato oggetto di numerosi studi. Tra gli altri si segnalano E. Sori, L'emigrazione italiana dall'Unità alla seconda guerra mondiale, Bologna, Il Mulino, 1979; Cent'anni di emigrazione italiana (1876-1976), Roma, Cser, 1978; P. Bevilacqua-A. De Clementi-E. Franzina, Storia dell'emigrazione italiana, Donzelli, 2001-2002, 2 voll.; Emigrazione italiana in America Latina, Roma, Ministero per i Beni e le Attività Culturali, Direzione Generale per gli Archivi, 2002, 2 voll.; M. Sanfilippo (a cura di), Emigrazione e storia d'Italia, Cosenza, Pellegrini editore, 2003.

${ }^{4} \mathrm{Mi}$ permetto qui di rimandare al mio Apprendere la concorrenza: l'emigrazione nei manuali scolastici europei tra la fine dell'Ottocento e i primi decenni del Novecento, in "Studi sulla Formazione", 2-2015, pp. 81-101.

${ }^{5}$ L. Giannitrapani, Compendio di geografia generale (fisica, biologica, umana) e nozioni di cosmografia, geografia matematica e geologica ad uso dei licei e degli istituti tecnici superiori, Firenze, R. Bemporad \& Figlio, 1925. Su Domenico Giannitrapani cfr. l'omonima voce di A. Pizzaleo in Dizionario Biografico degli Italiani, Volume 54 (2000) e S. Oliviero, Domenico e Luigi Giannitrapani geografi per la scuola, in G. Bandini (a cura di), Manuali, sussidi e didattica della geografia, cit., pp. 95-102.
} 
Giannitrapani, al pari di molti altri autori di manuali scolastici, spiega che a seconda delle modalità con cui viene messa in atto, l'emigrazione si distingue in "temporanea" e "permanente". Nel primo caso gli emigranti si stabiliscono in un determinato paese per compiere i lavori per un tempo limitato, magari anche solo in certi periodi dell'anno, e poi rientrano in patria. In tal caso, gli spostamenti possono verificarsi anche all'interno dello stesso Stato. Tuttavia, la forma più significativa di emigrazione è quella che avviene fra Stati diversi. Si tratta, allora, quasi sempre di emigrazione permanente perché i migranti partono per terre molto distanti, compiendo dalla terra natia un distacco che il più delle volte può risultare definitivo. Ciò avviene soprattutto in quegli Stati che hanno una crescita demografica costante, dove la popolazione in eccesso è costretta a cercare fonti di sussistenza al fuori dei confini patrii, come avviene per l'Italia, l'Inghilterra e la Germania.

Non sempre, però, tale distacco risulta nocivo alla madrepatria: infatti, se gli esuli sono un numero considerevole all'interno del nuovo Stato, possono formare una "colonia etnica o di popolamento", soggetta alla sovranità del Paese in cui risiedono. È questo il caso degli italiani che hanno colonizzato ampie porzioni dell'Argentina, del Brasile e della Tunisia: "se questi figli lontani dalla Madrepatria mantengono il ricordo della loro terra e continuano a parlare la loro lingua, le colonie etniche sono di grande vantaggio alla patria che manda in quei lontani paesi i suoi prodotti esuberanti e ne riceve denaro, diffonde per loro mezzo la sua lingua e la sua cultura ed aumenta così la propria importanza politica". 6

Come sottolinea Carmelo Colamonico, "dalle colonie etniche vanno considerate ben distinte le colonie più comunemente dette colonie politiche: per colonia politica $\mathrm{o}$ possedimento coloniale di uno Stato si intende il territorio su cui questo stato esercita un potere dominante; per solito il territorio dipendente è lontano dallo Stato sovrano (Libia). Se nel territorio dipendente vengono mantenute le organizzazioni statali indigene e i capi locali si tratta di protettorato (Tunisia). Quando poi si tratta di un territorio sul quale uno Stato, d'accordo con altri Stati, si è riservato il diritto ad una possibile futura espansione politico commerciale, si parla di sfera di influenza. Il paese coloniale, infine, il cui governo viene della Società delle nazioni temporaneamente affidato stato civile prende il nome di "mandato". 7

Nella sua dettagliata analisi del fenomeno migratorio, Giannitrapani spiega che le colonie variano anche di "carattere", poiché risentono delle attitudini dei popoli che emigrano: per esempio, le colonie italiane sono prevalentemente agricole perché popolate da contadini, quelle inglesi e tedesche sono per lo più commerciali perché composte da commercianti e industriali.

Spiegazioni analoghe sono offerte da Roberto Almagià nel suo Manuale di geografia ad uso delle scuole superiori e delle persone colte, anch'esso edito nel $1925 .{ }^{8}$ Almagià non si limita a illustrare in maniera astratta cause e modalità delle migrazioni, ma fornisce al lettore alcune indicazioni utili per capire quale sia la realtà con cui si confrontano, ad esempio, gli italiani che vanno a cercare fortuna negli Stati Uniti: lì svolgono i lavori più umili, "sono poco apprezzati e molto facilmente sfruttati; [...] arrivando senza conoscenze e senza mezzi, restano alla mercè di incettatori poco onesti. Contribuisce a peggiorare le condizioni

\footnotetext{
${ }^{6}$ Ivi, p. 456.

${ }^{7}$ C. Colamonico, Corso di geografia ad uso delle scuole medie superiori, Secondo i recenti programmi di insegnamento, Vol. I, La geografia generale e la geologia. Quinta edizione interamente rifatta, Milano, Vallardi, 1920, pp. 217-218.

${ }^{8}$ R. Almagià, Manuale di geografia ad uso delle scuole superiori e delle persone colte. Con numerose illustrazioni, carte e tavole fuori testo, Napoli- Genova-Città di Castello, F. Perrella, 1925, 2 voll. Su Almagià cfr., oltre alla voce a lui dedicata da I. Caraci Luzzana nel Dizionario Biografico degli Italiani, Volume 34 (1988), pp. 76-78, G. Corna-Pellegrini (a cura di), Roberto Almagià e la geografia italiana nella prima metà del secolo. Una rassegna scientifica e una antologia degli scritti (Atti del convegno tenuto a Milano nel 1986), Milano, Unicopli, 1988; vedi anche B. Vecchio, Geografia accademica e associazionismo geografico tra Otto e Novecento, in G. Bandini (a cura di), Manuali, sussidi e didattica della geografia, cit., pp. 22-26.
} 
l'ignoranza degli emigranti stessi, che non hanno sovente alcuna idea del paese ove si recano e del mestiere che vi faranno". 9

Insomma, per un'emigrazione di successo c'è bisogno di preparazione, anche scolastica, come sosteneva già nel 1888 Gerolamo Olivati nel suo Manuale di geografia cosmografica, fisica e politica ad uso delle scuole secondarie, classiche, tecniche, militari e navali, secondo cui è indispensabile "avere cognizioni sicure e pratiche, sia d'agricoltura, che di commerci o d'industrie; avere almeno un principio di cultura intellettuale (e moltissimi dei nostri contadini sono pur troppo analfabeti) e conoscere la lingua del paese. Altrimenti si va incontro alla sorte più lagrimevole, a patimenti infiniti, e senza frutto!". ${ }^{10}$

Se esistono, quindi, motivi strettamente biografici e familiari per evitare di emigrare, quelli più cogenti dovrebbero certamente essere di natura patriottica. Infatti, quasi tutti gli autori presi in considerazione condividono il giudizio di Almagià, secondo cui 'l'emigrazione permanente è un grave danno per l'Italia, perché sottrae durevolmente alla patria gli individui più attivi e vigorosi, lasciando quelli improduttivi (vecchi, donne, fanciulli); inoltre rilascia i vincoli della famiglia e l'affetto del paese. [...] In complesso questo dell'emigrazione è uno dei più grossi e difficili problemi dell'Italia moderna e torna sin d'ora ad imporsi in tutta la sua gravità, poiché terminato il conflitto mondiale, molti di coloro che erano rimpatriati per la guerra, nuovamente rivalicano l'Oceano". ${ }^{11}$

Al contrario, "l'espansione coloniale è oggigiorno un bisogno degli stati più importanti della terra; essa, infatti, oltre che dallo stimolo ad accrescere considerevolmente il prestigio di cui si gode nel mondo, è sorretta dalla necessità di trovare uno sbocco alla popolazione in costante aumento della madrepatria e dalla necessità di rifornire di materie prime l'industria in crescente sviluppo nella madrepatria. Queste necessità sono spiccatamente sentite del nostro paese in cui, nonostante la diminuita intensità degli ultimi anni, la popolazione è sempre in considerevole aumento e diventa sempre più notevole la sua attività industriale. L'Italia ha per di più una tradizione millenaria di potenza colonizzatrice. Nessun altro paese quindi può vantare maggiori diritti dell'Italia ad un allargamento del dominio coloniale". ${ }^{12}$

\section{Emigrazione e colonizzazione. La popolazione come ricchezza nazionale}

Indipendentemente dall'attenzione riservata al tema, i manuali di geografia prodotti in Italia tra la fine dell'Ottocento e i primi decenni del Novecento fanno riferimento a un comune bagaglio ideologico, politico e culturale per spiegare le relazioni tra l'Europa e gli altri continenti, oltre che tra la "civiltà" occidentale e quelle delle altre parti del mondo. Il fenomeno migratorio rappresentava, infatti, uno solo dei molteplici aspetti delle politiche internazionali degli Stati europei, impegnatissimi all'epoca a contendersi il controllo commerciale e militare degli altri continenti. Senza dimenticare che la politica coloniale li metteva in competizione gli uni con gli altri, dando vita a una gara in cui la popolazione, la crescita industriale, le colonie e l'alfabetizzazione rappresentavano tutti fattori fondamentali nel decidere a chi spettasse la supremazia politica ed economica nel Vecchio e nel Nuovo Mondo.

In quanto sapere consustanziale alla costruzione e alla diffusione di un'idea sufficientemente chiara del nostro pianeta e del ruolo svolto su di esso dalla nostra specie, la geografia si sforzò all'epoca di contribuire a fornire una nuova lettura della realtà, sia come

\footnotetext{
${ }^{9}$ Ivi, vol. 2, p. 312.

${ }^{10}$ G. Olivati, Manuale di geografia cosmografica, fisica e politica ad uso delle scuole secondarie, classiche, tecniche, militari e navali. $6^{\circ}$ edizione arricchita di molte figure e interamente riveduta e modificata dal dott. Giuseppe Marina, professore nel R. Liceo di Livorno, Livorno, R. Giusti, 1899, libro 2, p. 497. Una breve ma esaustiva biografia di Gerolamo Olivati si trova nella "Rivista geografica italiana", Vol. 3, IX, p. 535.

${ }^{11}$ R. Almagià, Manuale di geografia ad uso delle scuole superiori e delle persone colte, cit., vol. 2, pp, $313-314$.

${ }^{12}$ C. Colamonico, Corso di geografia ad uso delle scuole medie superiori, cit., p. 218.
} 
scienza sia soprattutto come disciplina scolastica. Non a caso, dalla geografia fisica, economica e più raramente politica, come era stata presentata sino a tutti gli anni Sessanta dell'Ottocento, si passò alla geografia umana, introdotta ufficialmente nella scuola italiana dai programmi del 1888 e del 1894. Da quel momento, lo studio delle nazioni e delle loro forme di governo e di economia divenne gradualmente prioritario a scuola.

I nuovi orientamenti disciplinari risultarono funzionali ai bisogni degli Stati europei del periodo, la cui politica era incentrata sulla conquista coloniale e sulla scoperta e lo sfruttamento delle risorse dei Paesi meno sviluppati. In effetti, come sostiene Horacio Capel, "among the reasons that explain the triumph of geography over rival disciplines, there is one of great importance: the function assigned to geography in the shaping of a feeling of nationalism". ${ }^{13}$

In tal senso, anche gli uomini rientravano tra gli elementi di ricchezza di una nazione. Se la terra era prima di tutto terra di conquista, armata (come nel caso dell'Africa, dove si provò vanamente a mettere ordine - oggi sappiamo con quali effetti! - con la Conferenza di Berlino) o industriale e commerciale, gli uomini venivano intesi come "capitale umano", in grado di rendere grande il Paese con il loro lavoro e il loro sapere. In questo contesto, gli abitanti delle nazioni europee, più ancora di quelli delle colonie, assunsero il ruolo di risorsa economica e politica fondamentale, dunque, da valorizzare e incrementare, prima di tutto per mezzo dell'istruzione, in base al principio che "chi più sa, più vale".

Proprio nella seconda metà dell'Ottocento divenne gradualmente condivisa l'idea secondo cui il valore di una popolazione non si calcola soltanto in termini numerici e quantitativi, sebbene essi rappresentino un aspetto fondamentale spesso evocato quando si parla di emigrazione. Conta anche il livello culturale o, per dirlo con le parole usate all'epoca, "di civiltà" di cui gli uomini sono portatori. Nella maggioranza dei casi, però, l'aspetto che per primo viene affrontato è quello dell'importanza della popolazione come risorsa materiale per la nazione: non è realizzabile nessuna forma di grandezza nazionale senza una popolazione numerosa e fertile. Poi, certo, nella competizione internazionale è fondamentale anche il livello d'istruzione dei cittadini, ma il primo requisito che determina l'importanza di un Paese è senza dubbio il numero dei suoi abitanti.

Un requisito che l'Italia dell'epoca possedeva certamente a differenza di altri Paesi vicini e concorrenti, come la Francia, che nello stesso periodo sperimentava un significativo calo di abitanti dopo secoli di crescita. Nonostante il saldo demografico ampiamente positivo, gli autori italiani manifestavano grande rammarico per la perdita di capitale umano causata dagli ingenti flussi migratori, anche se non potevano fingere di ignorare che abbandonare la terra d'origine era spesso l'unica scelta possibile per i loro connazionali. Almagià, ad esempio, afferma che 'l'emigrazione permanente è un grave danno per l'Italia, perché sottrae durevolmente alla patria gli individui più attivi e vigorosi, lasciando quelli improduttivi (vecchi, donne, fanciulli); inoltre, rilascia i vincoli della famiglia e l'affetto del paese. Gli emigranti sono infatti quasi soltanto adulti nel fior dell'età e per lo più partono soli, lasciando qui la famiglia. Non si deve però disconoscere che l'emigrazione è una fonte di guadagno, perché gli emigranti che fanno ritorno, portano in patria il frutto del proprio lavoro e se ne servono per migliorare le proprie condizioni; inoltre, ritornano più intraprendenti, più temprati al lavoro. [...] $\mathrm{Ma}$ in complesso questo dell'emigrazione è uno dei più grossi e difficili problemi dell'Italia moderna e torna sin d'ora ad imporsi in tutta la sua gravità, poiché terminato il conflitto mondiale, molti di coloro che erano rimpatriati per la guerra, nuovamente rivalicano l'Oceano". ${ }^{14}$

\footnotetext{
${ }^{13}$ H. Capel, Institutionalization of Geography and Strategies of Change, in D. R. Stoddart (ed.), Geography, Ideology and Social Concern, Cambridge, Basil Blackwell, 1982.

${ }^{14}$ R. Almagià, Manuale di geografia ad uso delle scuole superiori e delle persone colte, cit., vol. 2, pp. 313-314.
} 
Il vero danno per la nazione derivava proprio dall'emigrazione cosiddetta "permanente", ovvero di quei concittadini che non solo non facevano più ritorno in patria, ma che si integravano perfettamente nella nuova realtà di vita. Per questo motivo, il Testo atlante scolastico di geografia moderna astronomica - fisica - antropologica espressamente compilato e disegnato per le scuole secondarie italiane del 1897, sottolineava con rammarico che "il nostro popolo, dopo qualche tempo che si è stabilito nei suddetti paesi [del Sud America], tende a confondersi col resto della popolazione e i loro figli dimenticano la lingua della madre patria per adottare quella del paese in cui dimorano", a differenza, per esempio, dei discendenti dei coloni francesi, che conservano la loro identità, la religione e la lingua d'origine, facendone: "un distintivo nazionale e una ragione di autonomia". 15

Del resto, era questo uno dei prezzi da pagare, come ricordava Battista Vay in un passaggio molto retorico e commuovente dell'Immagine del mondo per la quarta classe, per "il genio e le braccia" italiani, che con i loro sacrifici e la loro forza avevano permesso lo sviluppo di paesi altrimenti selvaggi, come l'Argentina. ${ }^{16}$

Vitale era, quindi, conservare un legame con gli emigranti, qualora costoro non tornassero in patria. Era questo l'unico modo per trarre un beneficio economico dalla migrazione, espandere l'influenza culturale e politica della nazione o quantomeno conservare un legame e una certa influenza - con quanti lasciavano la madrepatria per raggiungere terre lontane e spesso non ancora dotate di un forte senso di nazione, oltre che di "civiltà".

\section{Emigrazione italiana e Sud America}

Il tema dell'emigrazione nel dibattito politico coevo e, di conseguenza, nei manuali, era strettamente legato a quello della colonizzazione, ovvero della conquista militare e dell'influenza economico-politica sui Paesi extra-europei. Questo approccio al tema dell'emigrazione era comune a tutti i Paesi europei, che all'epoca si scontravano sull'agone economico e politico, prima di arrivare a farsi la guerra sui campi di battaglia. ${ }^{17}$

In molti testi si sostiene ciò che con grande chiarezza spiega Almagià nel suo Manuale di geografia ad uso delle scuole superiori e delle persone colte, ovvero che "la tendenza di ogni popolo e nazione è quella di espandersi. Gli effetti prodotti da tale espansione sono la colonizzazione e l'emigrazione. Nel primo caso i territori esterni vengono occupati e legati politicamente allo stato occupante, nel secondo una parte dei cittadini abbandona la madrepatria per stabilirsi in terra straniera temporaneamente o definitivamente: in tal modo si costituiscono le colonie etniche o di popolamento". ${ }^{18}$

${ }^{15}$ A. Ghisleri-G. Roggero-G. Ricchieri, Testo atlante scolastico di geografia moderna astronomica - fisica antropologica espressamente compilato e disegnato per le scuole secondarie italiane in conformità dei programmi governativi e delle moderne esigenze pedagogiche. Fascicolo IV: Asia, Oceania, Africa, America e terre polari. Edizione per le scuole secondarie superiori (ginnasiali, istituti tecnici e normali), Bergamo, Istituto di arti grafiche, 1897, p. 98. Su Ghisleri cfr. F. Maffei, La formazione di un geografo. Arcangelo Ghisleri e il rinnovamento degli studi geografici in Italia (1878-1898), Pisa, ETS, 2007.

${ }^{16}$ B. Vay, L'immagine del mondo. Corso di geografia per le scuole elementari. Quarta classe, Firenze, Vallecchi, 1925. I manuali di geografia per le scuole elementari di Vay furono tra i più apprezzati dalla commissione ministeriale del 1926. Cfr. A. Ascenzi-R. Sani, Il libro per la scuola tra idealismo e fascismo. L'opera della Commissione centrale per l'esame dei libri di testo da Giuseppe Lombardo Radice ad Alessandro Melchiori (1923-1928), Milano, Vita \& Pensiero, 2005, p. 619 e M. Galfré, Il regime degli editori. Libri, scuola e fascismo, Bari, Laterza, 2005, p. 25.

${ }^{17}$ Cfr. G. Gabrielli, Il curricolo razziale. La costruzione dell'alterità di "razza" e coloniale nella scuola italiana (1860-1950), Macerata, EUM, 2015; Id., Educati alla guerra. Nazionalizzazione e militarizzazione dell'infanzia nella prima metà del Novecento, Verona, Ombre corte, 2016. Mi permetto di rimandare anche a P. Bianchini, Apprendere la concorrenza: l'emigrazione nei manuali scolastici europei tra la fine dell'Ottocento e $i$ primi decenni del Novecento, in "Studi sulla Formazione", 2-2015, pp. 81-101.

${ }^{18}$ R. Almagià, Manuale di geografia ad uso delle scuole superiori e delle persone colte, cit., vol. 1, p. 407. 
Nei manuali di geografia, i temi dell'emigrazione e della colonizzazione sono più o meno implicitamente collegati all'"istinto" di espansione dell'uomo, in particolare della razza bianca. Per questo motivo, Almagià, al pari di molti altri autori scolastici, sottolinea con una certa enfasi e un certo orgoglio che "si calcola che nell'ultimo secolo l'Europa abbia inviato di là dell'Oceano oltre 30 milioni di suoi figli"" e che "nessun paese d'Europa dà tanti emigranti quanti l'Italia". ${ }^{20}$

Archetipo dello Stato europeo votato alla colonizzazione è senza dubbio l'Inghilterra. Anche Luigi Hugues, nei suoi fortunatissimi Elementi di geografia ad uso delle scuole secondarie, commerciali e militari, indica come principale caratteristica dell'Europa proprio i possedimenti che numerosi Stati detengono negli altri continenti o nel proprio (ad esempio, Gibilterra, geograficamente spagnola, ma appartenente all'Impero Britannico). E per offrire agli studenti un esempio concreto della capacità europea di espandersi, Hugues fa riferimento al "popolo inglese", definito dall'autore "eminentemente colonizzatore". ${ }^{21}$

Tuttavia, se emigrazione e colonizzazione sono due facce della stessa medaglia, l'espatrio a titolo definitivo verso Paesi stranieri rappresenta senza dubbio un aspetto negativo per la madrepatria. Infatti, come abbiamo già visto, quando un europeo valica mari o oceani per andare a stabilirsi in qualche terra lontana indebolisce la sua nazione d'origine e reca, invece, un indubitabile beneficio per quella di destinazione.

Per questi motivi, quando i manuali offrono consigli su dove emigrare, indicano naturalmente i luoghi in cui è già forte la presenza europea. Dunque, se proprio si decide di lasciare il suolo patrio, vale la pena di farlo in direzione delle colonie. Qualora le colonie non siano in grado di ricevere nuova popolazione o si decida di cercare fortuna voltando le spalle alla patria, conviene rivolgersi al continente americano e, in particolare, al Sud America, dove le possibilità di compiere un'emigrazione di successo restano più elevate che altrove.

In realtà, neanche tutti i Paesi sudamericani si equivalgono, così come non tutti i migranti si assomigliano. Se Paesi giovani, ma meta da tempo della colonizzazione europea, possono dirsi ben avviati sulla strada della civilizzazione, come l'Argentina, l'Uruguay e il Cile, altri come il Brasile sono descritti come ricchissimi di opportunità, ma quasi del tutto selvaggi. Come illustra in maniera dettagliata Guido Branca nella sua Geografia elementare proposta alle scuole primarie, molti degli stati nei quali è diviso il Brasile "sono percorsi ancora da orde selvagge e la scienza le conosce appena nelle caratteristiche più generali dagli itinerari di pochi viaggiatori le hanno attraversate fra stenti e pericoli. L'emigrazione europea accresce bensì ogni anno la popolazione ma assai lentamente; il commercio marittimo va crescendo, s'incoraggiano le industrie e gli studi, si frena per quanto possibile l'immoralità dei costumi agevolata dalla mistione delle razze e dall'ignoranza". ${ }^{22}$

Poco importa se gli emigranti italiani vanno spesso incontro a sofferenze dovute allo sfruttamento. Autori come Giannitrapani sono ben consapevoli del fatto che in Brasile "nel 1888 essendo stata abolita la schiavitù dei negri, quei terreni rimasero quasi senza lavoratori e fu necessario richiamare gli emigranti europei, fra il quali accorsero in gran numero gli

\footnotetext{
${ }^{19}$ Ivi, vol. 1, p. 166.

${ }^{20}$ Ivi, vol. 1, p. 408.

${ }^{21}$ L. Hugues, Elementi di geografia ad uso delle scuole secondarie, commerciali e militari. Primo corso di geografia generale. Secondo corso di geografia dell'Europa. Terzo corso di geografia dell'Asia, Africa, Australia e Polinesia, America, Terre Polari, Torino, E. Loescher, 1891, VII ed., p. 121. Su Luigi Hugues, oltre alla voce a lui dedicata da N. Fusco e M. T. Dellaborra in Dizionario Biografico degli Italiani, Vol. 61 (2004), cfr. I. Luzzana Caraci, La geografia italiana tra '800 e '900 (dall'Unità a Olinto Marinelli), Genova, Istituto di Scienze Geografiche della Facoltà di Magistero, 1982, pp. 13 e segg.; G.M. Mongini, La didattica della geografia nei primi decenni dell'Italia unita, in “Bollettino della Società geografica italiana”, s. 11, VI (1989), 1, pp. 47-69.

${ }^{22}$ G. Branca, Geografia elementare proposta alle scuole primarie. Seconda edizione riveduta ed accresciuta dal prof. Guido Cora, Torino, Loescher, 1872, p. 144.
} 
italiani che sono ora circa 1.500 .000 e sono stabiliti come salariati nelle fazende, oppure come coloni indipendenti". ${ }^{23}$

Non sempre, l'avventura e la scarsa concorrenza sono garanzie sufficienti di successo. Al contrario, la presenza di compatrioti e di una nutrita colonia di europei spinge gli autori dei manuali a invitare i loro giovani connazionali che intendono andare Oltreoceano a preferire l'Argentina o l'Uruguay al Brasile, in quanto vi si può trovare facilmente qualcosa di simile a ciò che si è lasciato in patria. Anche in quei casi, tuttavia, occorre preparare in maniera accorta il proprio viaggio per rendere l'emigrazione davvero produttiva per sé e per la madrepatria.

Trattando dell'Argentina, sempre Giannitrapani spiegava che il milione e mezzo di italiani che vi risiedono "esplicano una grande attività commerciale e prendono parte attiva alle iniziative di lavori pubblici ed agricoli e a speculazioni bancarie. Ma tali attività ed iniziative conservano un carattere individualistico in questa come nelle altre nostre colonie di emigrazione; sicché, mancando l'espressione della vita e della forza collettiva che esplicano invece le colonie di altre nazioni, gli italiani hanno scarsa influenza sulla vita pubblica del paese. L'Argentina è il paese che attualmente richiama la maggior parte della nostra emigrazione transoceanica perciò questa colonia e destinata ad uno sviluppo sempre maggiore". ${ }^{24}$

Allo stesso modo, dopo aver spiegato che per un'emigrazione di successo in paesi sviluppati come gli Stati Uniti è necessario avere un buon livello d'istruzione, Almagià insiste sulla necessità di scegliere con cura il paese in cui migrare, in quanto l'esperienza che gli italiani hanno accumulato in materia dimostra che "nel Brasile vengono impiegati nelle piantagioni di caffè dove sono sottoposti a fatiche ben più pesanti rispetto a quelle che sostengono nel suolo natio come contadini, condizionati da un clima poco favorevole agli europei e senza ricevere compensi adeguati. In Argentina e in Uruguay le condizioni sono assai migliori poiché lavorano in un clima affine a quello della madrepatria e si occupano delle stesse colture presenti in Italia". ${ }^{25}$

Molti manuali spiegano che in Paesi come l'Argentina, l'Uruguay e il Cile, l'emigrante italiano può trovare condizioni simili a quelle che ha lasciato in Europa, ideali per integrarsi e realizzarsi: "A cagione della migliore situazione, specie nelle province pre-andine formano grandi colonie agricole, i cui nomi si ispirano alle città e ai paesi italiani. Un numero considerevole vive anche nei centri urbani dove esercitano mestieri, arti liberali e professioni: molti di loro sono apprezzati e hanno saputo conquistarsi posizioni illustri". ${ }^{26}$ Non a caso, il maggior numero di connazionali si trova in Argentina, la quale rappresenta: "il più grande centro dell'emigrazione italiana in America". Le cifre stimano che dal 1857 al 1913 si siano stabiliti circa 2.000.000 di italiani e nel 1913 pare ve ne fossero presenti 850.000. Altre numerose colonie di italiani prosperano nel Brasile e nell'Uruguay". ${ }^{27}$

Se il Brasile non condivide con le colonie spagnole e inglesi una situazione di relativa uscita dalla barbarie e di pieno sfruttamento del suo enorme potenziale è colpa, secondo Gerolamo Boccardo, compilatore di fortunatissimi manuali scolastici, dell'impronta lasciata dalla madrepatria, che "quando nel 1654 ricuperò il Brasile, si occupò con tarda sollecitudine dell'unico stabilimento coloniale che del suo vasto impero rimanesse." Per questo, "il

${ }^{23}$ L. Giannitrapani, L'Italia e le sue colonie. Manuale di geografia ad uso dei ginnasi inferiori, Secondo $i$ programmi ministeriali del 20 ottobre 1923, con 181 illustrazioni e 4 carte geografiche a colori, Firenze, Bemporad \& figlio, 1924, p. 290.

${ }^{24}$ Ivi, p. 291.

${ }^{25}$ R. Almagià, Manuale di geografia ad uso delle scuole superiori e delle persone colte, cit., vol. II, p. 312.

${ }^{26}$ Ibid.

${ }^{27}$ E. Comba, Compendio di geografia teorico-pratica con notizie storiche ad uso delle scuole ginnasiali, normali, tecniche, industriali e commerciali. Nuova ristampa riveduta dal prof. Carlo Errera. Opera adottata con autorizzazione del Ministero della Pubblica Istruzione, Torino, G. B. Paravia e Comp., 1913, p. 313. 
Portogallo non si rialzò più mai dall'abbiezione in cui era caduto. L'indole neghittosa degli abitanti li teneva lontani dal lavoro e dall'industria. Lo spirito di restrizione e di monopolio esauriva le fonti della ricchezza ed incagliava il commercio. La brama di far pronta fortuna nelle miniere rimuoveva dalle vie più produttive la popolazione". ${ }^{28}$

Per l'Argentina in generale il giudizio è diverso: essa, infatti, sotto alcuni punti di vista può essere assimilata a uno Stato europeo "grazie proprio agli immigrati, che rappresentano quasi la metà dei suoi otto milioni di abitanti, specialmente dall'Europa del sud, italiani in testa. Molti manuali sottolineano, non a caso, che "la repubblica Argentina è uno Stato in grande progresso", dotata di ferrovie, linee telegrafiche ed efficienti mezzi di comunicazione via terra e via mare. ${ }^{29}$

Merito di tale supremazia sugli altri Paesi sudamericani è senza dubbio la precoce immigrazione dall'Europa, e specialmente dall'Italia, dato che, come ricorda "dal 1857 (forse qualche anno più tardi) al 1908 la nostra emigrazione è scaglionata verso il Rio de la Plata come le forze di un esercito". 30

Del resto, nonostante una certa arretratezza, è innegabile che il Nuovo Mondo possegga una vitalità straordinaria: nelle Nozioni di geografia ad uso delle scuole tecniche di Niccolò Da Ponte si legge che la civiltà americana ha moltissimi tratti in comune con quella europea, in quanto vi è stata importata dagli emigrati, ma se ne distingue per "un certo slancio, una certa originalità e vitalità tutta propria del Nuovo Mondo". ${ }^{31}$ I giudizi ottimistici nei confronti del Sud America non sono rari all'interno dei testi di geografia e diventano, appunto, l'occasione per tessere le lodi dell'Europa colonizzatrice.

Se i giudizi sulle singole nazioni extra-europee sono comuni a buona parte dei manuali editi in Italia (così come in altri paesi europei) tra la fine dell'Ottocento e l'inizio del Novecento, è perché è quasi identica la rappresentazione complessiva del pianeta, presentato ai giovani lettori come terra di conquista, un'opportunità che le potenze europee sono state 0 meno capaci di cogliere. Emerge un'idea dell'altro fortemente stereotipata, fatta di luoghi comuni, in cui ciò che viene sottolineato è, più che la distanza geografica, la differenza dalla normalità, rappresentata dall'Europa.

Non è raro, quindi, trovare ancora nei manuali di inizio Novecento giudizi e termini vecchi di secoli, non solo del tutto infondati, ma anche fuorvianti per gli studenti. Ad esempio, non sono infrequenti gli autori che continuano a definire il continente americano "Indie Occidentali", evocando l'epopea delle scoperte geografiche e delle prime conquiste coloniali. Alla luce di stereotipi di questo tipo non stupisce leggere, a proposito del continente americano, che "gli indigeni, che nel passato conobbero periodi di prosperità e grande civiltà, quale quella Azteca e quella Inca, sterminati dagli europei, rappresentano la minoranza della popolazione. Tra di loro una parte ha dimora stabile ed esercita l'agricoltura, l'altra è nomade e vive di pesca e caccia. L'elemento civilizzatore fu però introdotto dai popoli immigrati appartenenti alla razza bianca. Tali popoli, grazie al contributo specialmente degli inglesi,

${ }^{28}$ G. Boccardo, Manuale di storia del commercio delle industrie e dell'economia politica, Ad uso delle scuole speciali secondarie, Torino, tipografia scolastica di Sebastiano Franco e figli e compagnia, 1858, p. 190.

${ }^{29}$ L. Schiaparelli, Manuale completo di geografia e statistica compilato per uso delle famiglie, degl'istituti d'educazione privata e delle scuole classiche, tecniche, normali e magistrali del Regno d'Italia. Undecima edizione corredata di una carta cosmografica e coi dati statistici del 1870-71, Torino, Tommaso Vaccarino, 1871 , p. 388.

${ }^{30}$ G. Dainelli, Paesi e genti. Corso di geografia per le scuole medie inferiori, vol. IV, L'Asia, l'Africa, l'Oceania, l'America e le terre polari, Milano, Mondadori, 1930, p. 242. Il testo è tratto da un brano di G. Pavoni dal titolo Il nostro lavoro nell'Argentina coi primi migranti.

${ }^{31}$ N. Da Ponte, Nozioni di geografia ad uso delle scuole tecniche per Niccolò da Ponte, professore di storia e geografia nella Reale Scuola Giulio in Torino, VI edizione riveduta e corretta, Torino, Pozzo, 1895, p. 73. 
permisero agli Stati Uniti di raggiungere una importanza economica ed un grado di cultura equivalenti a quelli dei più grandi e civilizzati Stati europei". ${ }^{32}$

\section{La razza come fondamento biologico delle idee di patria e nazionalismo}

Nei manuali di geografia italiani della fine del XIX e dell'inizio del XX secolo l'immagine dei connazionali nei paesi colonizzati e d'immigrazione è sempre strettamente connessa con la presentazione del diverso, ovvero degli altri popoli e delle razze differenti da quella bianca. In realtà, la rappresentazione dell'altro è ciò che più evidentemente si è evoluto nel periodo preso in considerazione. Il discorso sulla superiorità della razza bianca è, infatti, presente sin dalla fine dell'Ottocento, ma all'epoca vive ancora una fase embrionale e, per così dire, ingenua. La superiorità dei popoli bianchi viene, infatti, giustificata soprattutto con la superiorità della civilizzazione europea. È proprio tale vantaggio culturale che autorizza, o quasi obbliga moralmente, le nazioni europee a espandersi a danno di tutte le altre, le quali, a loro volta, non possono che trarre beneficio da una tanto feconda invasione. ${ }^{33}$

In effetti, la totalità dei manuali presi in esame, al momento di descrivere le varietà umane presenti sul globo terrestre, si serve di classificazioni. Non bisogna dimenticare che la distinzione in categorie ben definite era uno dei tratti distintivi delle scienze positivistiche, comprese quelle umane come la medicina, la psichiatria, la sociologia, nonché la pedagogia. Negli ultimi decenni dell'Ottocento, tuttavia, l'elemento somatico e biologico era uno solo degli elementi utilizzati dai geografi per spiegare l'eterogeneità delle culture e dell'essere umano. Come spiega bene Plinio Cortesi "l'uomo può considerarsi relativamente al suo colore, alla religione che professa, al grado di cultura" ${ }^{34}$ In relazione al colore della pelle vengono comunemente distinte cinque razze, ovvero quella "bianca o caucasica", quella "negra o etiopica", quella "gialla o mongolica", quella "rosso-bruna o americana" e quella "olivastra o malese". Invece, in merito alla religione, "ovverosia al diverso modo onde il genere umano manifesta il sentimento religioso, gli uomini possono riassumersi in due grandi classi, cioè in quelli che riconoscono un solo Dio, che diconsi perciò "monoteisti", ed in quelli che ammettono e prestano il loro culto a più dei e che si appellano "politeisti". ${ }^{35}$ Esiste, poi, un'ultima distinzione, quella derivante dallo "sviluppo delle facoltà intellettuali e morali", la quale porta a suddividere gli uomini in "civili", "barbari" e "selvaggi". Possono dirsi "civili quando professino una religione, abbiano gentili costumi, letteratura, arti, leggi e governo; barbari quando non conoscano scritture o lettere, quantunque non manchino di istituzioni che li ravvicinino alle genti incivilite; selvaggi quando abbiano facoltà intellettuali poco sviluppate, quando traggano la vita in pochissima relazione cogli altri uomini e che per conseguenza poco o nulla conoscano le arti più necessarie alla vita". ${ }^{36}$

Altri autori, come Francesco Borrelli, compilatore del Compendio di geografia, introducono una quarta classificazione delle società umane, ovvero quella che tiene conto delle lingue che esse parlano, fornendo una descrizione ancora differente della molteplicità di culture. $^{37}$

32 D. Giannitrapani, Geografia per le scuole secondarie. Seconda edizione accuratamente riveduta ed ampliata, Firenze, R. Bemporad \& Figlio, 1896, p. 119.

${ }^{33} \mathrm{Su}$ questi temi cfr., tra gli altri, G. Bandini, Rappresentazioni della nazione e razzismo nella geografia scolastica tra Otto e Novecento, in Id. (a cura di), Manuali, sussidi e didattica della geografia, cit., pp. 53-70.

${ }^{34} \mathrm{P}$. Cortesi, Compendio di geografia matematica e fisica con particolare riflesso alle scuole nautiche, Fiume, Stabilimento tipo-litografico di Emidio Mohovich, 1974, p. 59.

${ }^{35}$ Ivi, pp. 59-60.

${ }^{36}$ Ivi, pp. 60-61.

${ }^{37}$ F. Borrelli, Compendio di geografia ordinato secondo i programmi governativi per le classi elementari superiori e per le scuole tecniche e normali ( $1^{\circ}$ anno), Torino, Tipografia di Giovanni Borgarelli, 1877. 
In generale, comunque, tutti gli autori condividono il giudizio di Schiaparelli, secondo cui la razza bianca è quella che "domina su tutti gli altri anche per la superiorità dell'intelligenza". ${ }^{38}$

Allo stesso modo, molto comune nei manuali dell'epoca è la tesi di Luigi Giannitrapani, il quale sostiene che la "razza europea, chiamata anche mediterranea, perché la sua attività, nella antichità e nel Medio Evo, si svolse nel bacino del mediterraneo, e altresì detta bianca o caucasica, [...] risulta superiore alle altre per aver sviluppato maggiormente qualità fisiche, morali ed intellettuali che le hanno dato la prevalenza nel mondo. ${ }^{39} \mathrm{Il}$ "popolo europeo", infatti, deve essere considerato come "il popolo più civile, poiché meglio di altri è stato capace di adattarsi e modificare l'ambiente circostante. Favorito dalle condizioni geografiche, ha saputo sviluppare qualità eccezionali, come intelligenza, perseveranza, iniziativa e previdenza per l'avvenire che gli sono servite per progredire costantemente, per consolidare i risultati raggiunti e per espandersi oltre i confini". ${ }^{40}$

La supposta supremazia degli europei viene decantata anche dal punto di vista estetico, come nel caso degli Elementi di geografia estesi anche ai programmi governativi per le scuole ginnasiali, tecniche, normali e magistrali di Giovanni Bindoni (1877), che definiscono la razza bianca come quella che "si distingue dalle altre per leggiadria di forme e maggiore sviluppo intellettuale". ${ }^{41}$ E sebbene "secondo il grado di civiltà o di coltura i popoli si dividano in civili, barbari e selvaggi" la supremazia "caucasica" non ha ancora ragioni biologiche. $^{42}$

Ciò che è universalmente condiviso dai manuali dell'epoca è che il colonialismo deve essere considerato come una diretta conseguenza della superiorità culturale dei bianchi, la cui civiltà è intrisa di spirito d'intraprendenza e di conquista. Essa può avere naturalmente avuto qualche effetto negativo sulle altre civiltà, ma si è rivelata nella maggior parte dei casi feconda ed ha contribuito all'uscita dalla barbarie degli altri popoli.

Lo dice bene, tra gli altri, Wilhelm Pütz, tradotto da Amato Amati, nella Guida allo studio della geografia comparata ad uso delle scuole classiche e tecniche, quando sostiene che una "peculiarità della popolazione europea è stata la capacità di coniugare le arti e le scienze con le attività" industriali e commerciali, che ha permesso loro, sebbene l'Europa rappresenti una piccola porzione del mondo, di ottenere "una superiorità morale su tutte le altre", concretizzatasi attraverso il dominio coloniale, che si è esteso "sopra una terza parte della superficie terrestre ed abbraccia quasi la metà del genere umano". 43

I modelli interpretativi a cui fanno esplicitamente riferimento i manuali sono spesso assai datati e vanno dall'illuminista Johann Friedrich Blumenbach, fisiologo e naturalista tedesco, autore di una classificazione craniometrica, al biologo e naturalista Georges Cuvier (autore, tra gli altri delle Leçons d'anatomie comparée) e al pedagogista francese ThéophileSébastien Lavallée, curatore delle opere di Madame de Maintenon e autore dell'aggiornamento della geografia di Malte-Brun. Su queste fonti si innestavano, poi, l'evoluzionismo e il positivismo applicati alla storia del genere umano, sulla scia di autori come Darwin e Durkeim, originando una lettura della civilizzazione in cui il metro di paragone era la cultura europea, considerata come quella più avanzata, nei confronti della

\footnotetext{
${ }^{38}$ L. Schiaparelli, Manuale completo di geografia e statistica, cit., p. 51

${ }^{39}$ L. Giannitrapani, Compendio di geografia generale, cit., p. 120.

${ }^{40}$ D. Giannitrapani, Geografia per le scuole secondarie, cit., p. 121.

${ }^{41}$ G. Bindoni, Elementi di geografia estesi anche ai programmi governativi per le scuole ginnasiali, tecniche, normali e magistrali, Settima edizione, Treviso, Tipografia di Luigi Zoppelli, 1877, p. 37.

${ }^{42}$ Ivi, p. 38.

${ }^{43}$ G. Putz, Guida allo studio della geografia comparata ad uso delle scuole classiche e tecniche di G. Putz. Nuova versione con miglioramenti ed aggiunte ed una breve descrizione dell'Italia del prof. Amato Amati, Preside del Reale Ginnasio e Liceo P. Sarpi, Milano, P. Carrara, 1872, p. 91. Su Amato Amati cfr. la biografia redatta da R. De Felice nel Dizionario Biografico degli Italiani, vol. 2 (1960), pp. 669-670.
} 
quale gli altri popoli si ponevano a maggiore o minore distanza. Ci sarebbero voluti gli studi etnografici e antropologici, come quelli di Richard Thurnwald, per smantellare, non senza grande fatica e mai completamente, i presupposti ideologici dell'interpretazione europeocentrica della storia. ${ }^{44}$

L'interpretazione del mondo incentrata sulla civiltà e sul progresso, tuttavia, concedeva ai popoli extra-europei la possibilità di civilizzarsi, esattamente com'era successo ai bianchi. Era, insomma, una lettura non deterministica, dinamica, seppur funzionale agli interessi politici ed economici dell'Europa coloniale. Tale lettura consentiva a Colamonico di sostenere che " $\mathrm{i}$ bianchi ed $\mathrm{i}$ gialli formano le due razze più attive, più intelligenti e più numerose: hanno creato le forme più elevate di civiltà hanno imposto il loro dominio o la loro tutela a tutto il resto dell'umanità". ${ }^{45} \mathrm{E}$ lo stesso modo di pensare suggerisce al già citato Schiaparelli di affermare nel suo Manuale completo di geografia e statistica che, sebbene "il tipo negro" sia "inferiore agli altri due (il bianco e il giallo) in intelligenza e né mai produsse uomini ed opere veramente grandi, il che non significa ancora che non ne possa produrre". ${ }^{46}$

Ancora più esplicito era il Manuale di geografia moderna dell'inglese William Latham Bevan, tradotto in italiano da Giuseppe Carraro per conto dell'editore Barbera di Firenze. L'autore cominciava la trattazione sulla razza specificando che "la razza umana si mostra degna della posizione assegnatale nel mondo per la sua meravigliosa facoltà di adattarsi alle variazioni di clima, di alimentazione, di abitudine. Mentre gli animali sono confinati entro aree, qualche volta assai limitate, l'uomo può vivere e vive tanto sotto il sole equatoriale, quanto fra le nevi ed i ghiacci polari". ${ }^{47}$ Tali caratteristiche sono comuni a tutti gli esseri umani, in quanto "l'unità della razza è indubitata: havvi una sola specie con molte varietà elementari e le indagini etnologiche lo confermano. Le numerose e ben definite differenze di aspetto e di conformazione fisica quali sono di colore della pelle, la qualità dei capelli, la forma del cranio e l'angolo facciale, condussero a classificazioni delle varietà umane, che assai differiscono per il numero di esse varietà e per il principio su cui si basano". 48

Dunque, se ciò che caratterizza la razza bianca è che si è sviluppata più precocemente e meglio rispetto alle altre, ciò che varia nel corso del tempo, basandoci sull'analisi dei manuali presi in considerazione, sono le possibilità accordate alle altre razze di colmare tale differenza. Come spiega Giovanni Graziani, infatti, “in virtù dell'evoluzione, fondamento della biologia, certi gruppi umani, vivendo in ambiente adatto allo sviluppo fisiologico e psichico, si sono andati amalgamando, perfezionando e costituendo razze superiori. Altri gruppi, invece, vivendo in regioni poco favorite, o sono rimasti stazionari o sono andati invece a mano a mano decadendo, e si presentano oggi come popoli primitivi e rozzi". ${ }^{49}$

Gradualmente si comincia a spiegare la superiore dei popoli europei non solo con la civiltà, ma soprattutto con caratteristiche genetiche o comunque indissolubili dal contesto storico, geografico e culturale nel quale si è sviluppata la razza bianca. Tra i fattori considerati determinanti compare, nei primi del Novecento, il temperamento, che "è un carattere psichico importante, che si connette strettamente ai caratteri somatici, corrispondendo ciascuna razza

${ }^{44}$ S. J. Gould, Il geometra della razza, in Id., I Have Landed, Riflessioni di un naturalista sull'evoluzione, Torino-Roma, Codice Edizioni-Le Scienze, 2010, pp. 390-412.

${ }^{45}$ C. Colamonico, Corso di geografia ad uso delle scuole medie superiori, cit., vol. I, p. 203.

${ }^{46}$ L. Schiaparelli, Manuale completo di geografia e statistica, cit., p. 42.

47 W.L. Bevan, Manuale di geografia moderna, matematica, fisica e descrittiva di G.L. Bevan, Prima traduzione italiana con aggiunte e note ad uso degl'italiani. Terza edizione riveduta e corretta dall'autore e corredata di prospetti statistici, Firenze, Barbera, 1876, p. 60.

${ }^{48}$ Ibid.

${ }^{49}$ G. Graziani, La terra e le nazioni. Corso di geografia ad uso degli istituti tecnici e delle persone colte. Corredato da 207 illustrazioni e 5 tavole, nonché da scelte letture, Quarta edizione, Brescia, Giulio Vannini editore, 1923, pp. 210-211 
un temperamento spiccatamente particolare". Si scopre così che "proprio della razza mediterranea è il temperamento nervoso" dove "il sentimento e la ragione si moderano la vicenda ed, essendo oltremodo sviluppati, conferiscono a tale razza una decisa superiorità su tutte le altre. Invece, la razza mongoloide, dotata di un temperamento melanconico, tende per sua particolare disposizione d'animo a mirare più al passato che all'avvenire, per cui è conservatrice tenace delle tradizioni e dei propri costumi, e si equilibra in una stasi che, in tempi di progresso come i nostri, è sinonimo di regresso". ${ }^{50}$ Per questo, se anche le civiltà europee possono essere considerate quasi al passo con quelle occidentali, per il futuro la loro inferiorità sembra garantita, esattamente come per le altre razze: "la razza africana ha un temperamento sanguigno, è oltremodo impulsiva e mutevole nel pensiero e negli atti, non è facile a riflettere e non ha freni di inibizione. La razza americana, dotata di temperamento flemmatico, ha di particolare una imperturbabilità di animo che rende impassibile questa gente taciturna, e grave fin sotto l'azione del dolore fisico. Il temperamento collerico e caratteristico della razza malaica, appassionata, diffidente, crudele". ${ }^{51}$

Alla luce di questa disamina, la previsione di Graziani è che, poiché i popoli si distinguono in relazione al grado di civiltà raggiunta in naturali, semi-civili e civili, "oggi, nel fervore di una civiltà nuovissima, l'ultimo stadio evolutivo della attività umana sarebbe rappresentato dai popoli industriali e commerciali nei paesi dove (come nell'Inghilterra, nel Belgio, in parecchi distretti della Germania, degli Stati Uniti, della Francia) lo sviluppo dell'industria e dei traffici ha assunto una tale importanza da oltrepassare nel valore dei prodotti quella dell'agricoltura". ${ }^{5}$

In questo senso, la scomparsa dei popoli meno civilizzati può essere considerato, senza troppi rimpianti, come un danno collaterale dell'espansione della civiltà più progredita. Non sono pochi i manuali nei quali, sulla scia di Darwin e Durkeim, il passaggio dall'uomo selvaggio a quello "civile" viene presentato come una rinascita del genere umano. Ad esempio, Luigi Hugues, nei già citati Elementi di geografia ad uso delle scuole secondarie, commerciali e militari, per spiegare la strage di Maya e Atzechi, pur riconoscendo la notevole influenza di molteplici fattori, tra cui le guerre, le malattie importate dall'Europa e l'alcolismo, affermava che "ogni qualvolta le razze europee si trovano in contatto con una razza inferiore, questa decresce rapidamente, e finisce per scomparire senza lasciare di sé alcuna traccia apprezzabile". ${ }^{3}$

Ben piccolo prezzo da pagare se vogliamo credere a Eugenio Comba, secondo cui "le numerose colonie propagano la civiltà europea oltre i confini relativamente ristretti. [...] Perciò ben può dirsi, che l'Europa, nonostante la sua poca estensione di territorio, è la dominatrice di tutto il mondo, sopra il quale esercita la più benefica influenza in ogni ramo dell'umano incivilimento". 54

Alla luce delle differenze congenite tra le varie razze umane, lette con finalità teleologiche, la superiorità della razza bianca cessa di essere presentata come un prodotto culturale, e dunque, transitoria, per diventare, invece, perenne. Proprio nei decenni a cavallo del secolo, ma ben prima del successo dei totalitarismi, la superiorità della razza assunse motivazioni biologiche e meccanicistiche, con riferimenti etnici e quindi rigidamente immutabili. La questione della colonizzazione assume, così, un valore prima di tutto biologico e solo in un secondo momento culturale e politico, trovando spiegazione non con la politica o l'economia, ma con la natura stessa dell'uomo. E se la teoria della superiorità culturale

\footnotetext{
${ }^{50} I v i$, p. 216.

${ }^{51}$ Ivi, p. 217.

${ }^{52}$ Ivi, p. 223.

${ }^{53}$ L. Hugues, Elementi di geografia ad uso delle scuole secondarie, commerciali e militari, cit., p. 175.

${ }^{54}$ E. Comba, Compendio di geografia teorico-pratica con notizie storiche ad uso delle scuole, cit., pp. 56-57.
} 
concede alle altre razze la possibilità di colmare la distanza esistente con i bianchi, la supremazia basata sulla componente biologica rende incolmabile tale distacco.

Tra la fine dell'Ottocento e gli esordi del Novecento, ovvero ben prima della nascita del fascismo e del nazismo, in maniera estremamente rapida ed evidente per le tracce che ha lasciato nella letteratura scolastica, al giudizio sulla civiltà subentrò un giudizio sulla razza. Così, mentre la fisiognomica andò in aiuto dei geografi, a mano a mano che gli studi si susseguivano, la catalogazione dei "tipi" e dei "gruppi" umani divenne sempre più complessa. Il metro di paragone tra le razze non era più tanto la civiltà, quanto piuttosto l'angolo facciale, il temperamento, il clima, tutti fattori indissociabili da una determinata razza e dal suo ambiente.

Un ultimo passaggio logico, estremamente ardito, a cui vengono iniziati gli studenti europei di fine Ottocento-inizio Novecento è quello che indica il sentimento patrio come patrimonio esclusivo della razza bianca e della civiltà europea: non diversamente da quanto accade anche nel resto d'Europa, anche gli autori italiani concordano con Giannitrapani quando sostiene che "una caratteristica tipica dei popoli civili è il sentimento di nazionalità, che induce $i$ popoli a dotarsi di istituzioni politiche e civili per il mantenimento stesso dello stato e per la sua difesa". ${ }^{55}$ Lo stesso Giannitrapani sostiene che "gli europei sono tra i popoli più civili poiché, oltre a provvedere alle necessità materiali, tendono alla soddisfazione dei bisogni morali ed intellettuali. Tali popoli, in continua evoluzione, sono fonte di progressi nell'agricoltura, nell'industria, nelle scienze e nelle arti". In loro "è vivo il sentimento della nazionalità e si adoperano a mantenerla mediante le istituzioni politiche e la forza delle armi". ${ }^{56}$

Perfettamente in linea con la filosofia neo-idealista, prima ancora che con la politica fascista, gli autori di manuali di geografia italiani valutano il grado di civiltà di un popolo in base alla presenza o meno di un'idea di patria o di Stato, considerando l'assenza di un'organizzazione statale come la prova della condizione di arretratezza. Detto con altre parole, i libri di geografia si sforzano per mostrare agli studenti le relazioni tra la civiltà e il nazionalismo. Del resto, poiché le società non sono altro che l'evoluzione della famiglia e della tribù, i popoli "selvaggi" vengono presentati come "naturali", ovvero legati alle forme più basilari dell'organizzazione sociale, come la famiglia e la tribù. Al contrario, i popoli civili sono gli unici che hanno dato vita a forme statuali complesse sia dal punto di vista giuridico e costituzionale sia soprattutto per ciò che riguarda il sentimento patrio.

Il destino delle popolazioni meno civilizzate viene, per questi motivi, presentato come ineluttabile. Ne è un esempio l'ultimo capitolo del libro di lettura di Arnaldo Faustini, Orrori e meraviglie dell'universo, intitolato Come muore la razza umana. L'autore spiegava il problema dell'evoluzione della specie umana come un fenomeno che "si riduce anche dalle più lontane profondità della preistoria ad un avvicendarsi continuo, incessante e fatale di popoli che muoiono e di popoli che sorgono, come un fluttuar perenne di onde od un perenne rinnovellamento di spirito e di materia. [...] Noi vediamo così che la espansione territoriale o morale di un popolo va sempre a detrimento di un altro: il più debole, che allontanato e cacciato dai suoi domini o nei suoi domini soffocato, finisce per impoverirsi e, dall'impoverimento, per estinguersi. ${ }^{57}$ Faustini andava alla ricerca di "agonie umane" esemplari nel passato e nel presente e le individuava nei Mongoli di Gengis Khan, nei Lapponi, nei Pellerossa, nelle civiltà pre-colombiane, e più recentemente negli Aborigeni australiani e negli Onas della Terra del Fuoco (o Selk'nam) sopraffatti dai pionieri argentini e cileni, che hanno esaurito con la caccia la loro principale fonte di carne, il guanaco.

\footnotetext{
${ }^{55}$ L. Giannitrapani, Compendio di geografia generale, cit., p. 121.

${ }^{56}$ Ibid.

57 A. Faustini, Orrori e meraviglie dell'universo. Letture geografiche, Milano Roma Napoli, Società editrice Dante Alighieri di Albrighi, Segati \& C., 1912, pp. 293-294.
} 
Pur invitando il lettore a compiangere la sorte di quei popoli deboli e sfortunati, Faustini chiudeva il suo libro offrendo una lettura fatalista della loro estinzione, utile di fatto alla sopravvivenza del genere umano grazie agli esemplari più forti: "Sic transit gloria mundi. E nel perpetuo morire e rinnovellarsi delle cose di quaggiù è appunto tutta la filosofia della vita e dei popoli. E come gli abissi del mare e del cielo hanno i loro profondi e meravigliosi misteri, così gli abissi delle umanità, pur confusi nei più lontani confini della preistoria, hanno misteri non meno profondi e non meno meravigliosi”. 58

\section{RIFERIMENTI}

ALMAGIÁ. R. Manuale di geografia ad uso delle scuole superiori e delle persone colte. Con numerose illustrazioni, carte e tavole fuori texto. Napoli, Genova, Città di Castello: F. Perrella, 1925, 2 vol. https://doi.org/10.1038/102382a0

ASCENZI, A. e SANI, R. Il libro per la scuola tra idealismo e fascismo. L'opera della Commissione centrale per l'esame dei libri di testo da Giuseppe Lombardo Radice ad Alessandro Melchiori (1923-1928). Milano: Vita \& Pensiero, 2005.

BANDINI, G. (a cura di), Manuali, sussidi e didattica della geografia. Una prospettiva storica. Firenze: Firenze University Press, 2012.

BANDINI, G. I maestri e la geografia dopo l'unità d'Italia, in P. Bianchini (a cura di), Le origini delle materie. Discipline, programmi e manuali scolastici in Italia. Torino: SEI, 2010, p. 111-138.

BEVAN, W.L. Manuale di geografia moderna, matematica, fisica e descrittiva di G.L. Bevan, Prima traduzione italiana con aggiunte e note ad uso degl'italiani. Terza edizione riveduta e corretta dall'autore e corredata di prospetti statistici. Firenze: Barbera, 1876.

BEVILACQUA, P., DE CLEMENTI, A. e FRANZINA, E. Storia dell'emigrazione italiana. Roma: Donzelli, 2001-2002, 2 vol.

BIANCHINI, P. (a cura di). Le origini delle materie. Discipline, programmi e manuali scolastici in Italia. Torino: SEI, 2010.

BIANCHINI, P. Apprendere la concorrenza: l'emigrazione nei manuali scolastici europei tra la fine dell'Ottocento e i primi decenni del Novecento, in Studi sulla Formazione, 2-2015, pp. 81-101.

BINDONI, G. Elementi di geografia estesi anche ai programmi governativi per le scuole ginnasiali, tecniche, normali e magistrali, Settima edizione. Treviso: Tipografia di Luigi Zoppelli, 1877.

BOCCARDO, G. Manuale di storia del commercio delle industrie e dell'economia politica, Ad uso delle scuole speciali secondarie. Torino: Tipografia scolastica di Sebastiano Franco e figli e compagnia, 1858. https://doi.org/10.1038/102382a0

\footnotetext{
${ }^{58}$ Ivi, p. 295.
} 
BORRELLI, F. Compendio di geografia ordinato secondo i programmi governativi per le classi elementari superiori e per le scuole tecniche e normali $\left(1^{\circ}\right.$ anno). Torino: Tipografia di Giovanni Borgarelli, 1877.

BRANCA, G. Geografia elementare proposta alle scuole primarie. Seconda edizione riveduta ed accresciuta dal prof. Guido Cora. Torino: Loescher, 1872.

CAPEL, H. Institutionalization of Geography and Strategies of Change. In: STODDART, D. R. (ed.). Geography, Ideology and Social Concern. Cambridge: Basil Blackwell, 1982.

COLAMONICO, C. Corso di geografia ad uso delle scuole medie superiori, Secondo $i$ recenti programmi di insegnamento. Vol. I. Milano: Vallardi, 1920, p. 217-218.

COMBA, E. Compendio di geografia teorico-pratica con notizie storiche ad uso delle scuole ginnasiali, normali, tecniche, industriali e commerciali. Nuova ristampa riveduta dal prof. Carlo Errera. Opera adottata con autorizzazione del Ministero della Pubblica Istruzione. Torino: G. B. Paravia e Comp., 1913.

CORNA-PELLEGRINI, G. (a cura di). Roberto Almagià e la geografia italiana nella prima metà del secolo. Una rassegna scientifica e una antologia degli scritti (Atti del convegno tenuto a Milano nel 1986). Milano: Unicopli, 1988.

CORTESI, P. Compendio di geografia matematica e fisica con particolare riflesso alle scuole nautiche. Fiume: Stabilimento tipo-litografico di Emidio Mohovich, 1974.

DA PONTE, N. Nozioni di geografia ad uso delle scuole tecniche per Niccolò da Ponte, professore di storia e geografia nella Reale Scuola Giulio in Torino, VI edizione riveduta e correta. Torino: Pozzo, 1895.

DAINELli, G. Paesi e genti. Corso di geografia per le scuole medie inferiori, vol. IV, L'Asia, l'Africa, l'Oceania, l'America e le terre polari. Milano: Mondadori, 1930.

FAUSTINI, A. Orrori e meraviglie dell'universo. Letture geografiche. Milano, Roma, Napoli: Società editrice Dante Alighieri di Albrighi, Segati \& C., 1912.

FAVERO, L. e TASSELO, G. Cent'anni di emigrazione italiana (1876-1976). Roma: Cser, 1978.

GABRIELLI, G. Educati alla guerra. Nazionalizzazione e militarizzazione dell'infanzia nella prima metà del Novecento. Verona: Ombre Vorte, 2016.

GABRIELLI, G. Il curricolo razziale. La costruzione dell'alterità di "razza" e coloniale nella scuola italiana (1860-1950). Macerata: EUM, 2015.

GALFRÉ, M. Il regime degli editori. Libri, scuola e fascismo. Bari: Laterza, 2005. 
GHISLERI, A., ROGGERO, G., RICCHIERI, G. Testo atlante scolastico di geografia moderna astronomica - fisica - antropologica espressamente compilato e disegnato per le scuole secondarie italiane in conformità dei programmi governativi e delle moderne esigenze pedagogiche. Fascicolo IV: Asia, Oceania, Africa, America e terre polari. Edizione per le scuole secondarie superiori (ginnasiali, istituti tecnici e normali). Bergamo: Istituto di arti grafiche, 1897. https://doi.org/10.1038/102382a0

GIANNITRAPANI, D. Geografia per le scuole secondarie. Seconda edizione accuratamente riveduta ed ampliata. Firenze: R. Bemporad \& Figlio, 1896.

GIANNITRAPANI, L. Compendio di geografia generale (fisica, biologica, umana) e nozioni di cosmografia, geografia matematica e geologica ad uso dei licei e degli istituti tecnici superiori. Firenze: R. Bemporad \& Figlio, 1925.

GIANNITRAPANI, L. L'Italia e le sue colonie. Manuale di geografia ad uso dei ginnasi inferiori, Secondo i programmi ministeriali del 20 ottobre 1923, con 181 illustrazioni e 4 carte geografiche a colori. Firenze: Bemporad \& figlio, 1924.

GRAZIANI, G. La terra e le nazioni. Corso di geografia ad uso degli istituti tecnici e delle persone colte. Corredato da 207 illustrazioni e 5 tavole, nonché da scelte letture. Quarta edizione. Brescia: Giulio Vannini editore, 1923.

HUGUES, L. Elementi di geografia ad uso delle scuole secondarie, commerciali e militari. Primo corso di geografia generale. Secondo corso di geografia dell'Europa. Terzo corso di geografia dell'Asia, Africa, Australia e Polinesia, America, Terre Polari, Torino, E. Loescher, 1891, VII ed. https://doi.org/10.1038/067390a0

MAFFEI, F. La formazione di un geografo. Arcangelo Ghisleri e il rinnovamento degli studi geografici in Italia (1878-1898). Pisa: ETS, 2007.

OLIVATI, G. Manuale di geografia cosmografica, fisica e politica ad uso delle scuole secondarie, classiche, tecniche, militari e navali. $6^{\circ}$ edizione arricchita di molte figure e interamente riveduta e modificata dal dott. Giuseppe Marina, professore nel $R$. Liceo di Livorno. Livorno: R. Giusti, 1899, libro 2. https://doi.org/10.1038/102382a0

OLIVIERO, S. Domenico e Luigi Giannitrapani geografi per la scuola. In BANDINI, G. (a cura di), Manuali, sussidi e didattica della geografia. Firenze, Firenze University Press, 2012, p. $95-102$.

PUTZ, G. Guida allo studio della geografia comparata ad uso delle scuole classiche e tecniche di G. Putz. Nuova versione con miglioramenti ed aggiunte ed una breve descrizione dell'Italia del prof. Amato Amati, Preside del Reale Ginnasio e Liceo P. Sarpi. Milano: P. Carrara, 1872. https://doi.org/10.1038/067390a0

SANFILIPPO, M.(a cura di), Emigrazione e storia d'Italia. Cosenza: Pellegrini editore, 2003. 
SCHIAPARELLI, L. Manuale completo di geografia e statistica compilato per uso delle famiglie, degl'istituti d'educazione privata e delle scuole classiche, tecniche, normali e magistrali del Regno d'Italia. Undecima edizione corredata di una carta cosmografica e coi dati statistici del 1870-71. Torino: Tommaso Vaccarino, 1871. https://doi.org/10.1038/067390a0

SORI, E. L'emigrazione italiana dall'Unità alla seconda guerra mondiale, Bologna: Il Mulino, 1979.

TREMOLADA, C. La Geografia nei libri di testo per le scuole elementari (1859-1945), Tesi di dottorato, Università degli Studi di Milano, 1993. https://doi.org/10.15804/iw.2017.08.23

VAY, B. L'immagine del mondo. Corso di geografia per le scuole elementari. Quarta classe. Firenze: Vallecchi, 1925.

VECCHIO, B. Geografia accademica e associazionismo geografico tra Otto e Novecento. In: BANDINI, G. (a cura di). Manuali, sussidi e didattica della geografia. Firenze: Firenze University Press, 2012, p. 22-26. 